\title{
Versatility of Pectoralis Major High Splitting Technique in Breast Augmentation
}

\author{
MEDHAT S. HASSAN, M.D. \\ The Department of Plastic, Reconstructive \& Burn Surgery, Faculty of Medicine, Menoufia University, Egypt
}

\begin{abstract}
Breast augmentation is one of the most common aesthetic procedures required by plastic surgery female patients, and one of the most effective procedures changing their physical, psychological and social status as well. Certain problems would be corrected by breast augmentation as well such as amastia, hypoplastic breast, asymmetry, and age-related changes. To obtain an Ideal breast augmentation, there are some objectives that have to be fulfilled: The position of the nipples must be in the most projecting part of the breasts. The implant must be non-visible and non-palpable underneath the skin envelope. And the lower pole of the breast should be adequately filled than the upper pole. Thirty patients were included in this study using pectoralis major high splitting technique for the purpose of performing breast augmentation over the period of 5 years from February 2013 to November 2018. Follow-up period for each case was at least 6 months for the aesthetic outcome and patient satisfaction. Patients satisfaction was extremely high and there were no evidences for capsular contracture or abrupt demarcation of the implant under breasts skin. In conclusion performing breast augmentation using pectoralis major high splitting technique is of great value in giving a three-dimensional improvement in the continuously changing soft tissue dynamics of the breast, also decreases morbidity of the procedure and raises satisfaction and favorability of patients toward breast augmentation.
\end{abstract}

\section{INTRODUCTION}

Breast augmentation is one of the most common aesthetic procedures required by plastic surgery female patients, and one of the most effective procedures changing their physical, psychological and social status as well. But some patients would do augmentation to correct certain problems as amastia, hypoplastic breast, asymmetry, and agerelated changes [1].

To obtain an Ideal breast augmentation, there are some objectives that have to be fulfilled [2]:

1 - The position of the nipples must be in the most projecting part of the breasts.
2- The implant must be non-visible and nonpalpable underneath the skin envelope.

3- The lower pole of the breast should be adequately filled than the upper pole.

For that, various planes have been used to obtain these objectives such as: The subglandular [3] and total sub muscular [4], partial submuscular [10], dual [12] planes, each with its own advantages.

The variety of these planes was caused by the continuous changes happening to breasts by the factors of time and gravity. As its shape, size, and tissue quality vary and change with age, pregnancy, weight, and total body fat. This would bring back patients after a few years asking for correction of previous augmentation made by these various planes and techniques.

Some of the problems seen in sub glandular pockets are that a thin envelope would result in visible edges, increased palpability of the prosthesis, and traction rippling [3].

Partial or total sub muscular prosthesis placement in a thin individual usually gives an "unnatural" appearance of the breast in which there will be abnormal position of the nipples, high position of the prosthesis and a wide cleavage giving an artificial look which is not favored by the patient.

This happens because of the inadequate skin envelope limiting the descent of the implant, thus compromising the expansion of the skin envelope in a constricted lower pole of the breast. On the other hand, a submuscular implant with an excessive envelope usually results in a "double-bubble" appearance leaving the lower pole inadequately 
filled. Also the nipple position is not always achieved, resulting in a lack of three-dimensional improvement [4].

Muscle contraction induced deformities are common with submuscular implants [11] depending on the bulk and the length of the muscle fibers according to Frank-Starling Law [5] that states "energy of the contraction is proportional to the initial length of the muscle fiber." These distortions often are seen regardless of the division of the costal fibers of the pectoralis major.

Dual-plane breast augmentation [12] is the most widely used technique by plastic surgeons as it overcomes the problems seen in subglandular and submuscular planes.it is based on three criteria:

- The implant lies partially behind the pectoralis major muscle and partially behind the breast parenchyma (in dual planes simultaneously).

- A specific group of pectoralis major muscle origins is totally divided in a specific area.

- A change in the soft tissue relationship between the pectoralis major and parenchyma is performed in order to gain a better change in the implantparenchyma relation.

From these criteria we should have a pocket with an anatomic plane that can deal with the continuously changing dynamics of the breast. The procedure should allow quicker recovery for the patient, should be easier for the surgeon to perform as it is less invasive.

Pectoralis major high splitting augmentation is a procedure in which have similar goals to dual plane breast augmentation but with a more conservative and more anatomical purpose. As muscle fibers to be divided at the pectoralis major origin are markedly reduced, thus improving the morbidity and the recovery period for patients while fulfilling the objectives of an Ideal breast augmentation.

\section{PATIENTS AND METHODS}

This is a prospective study which was performed between February 2013 till November 2018 at Plastic Surgery Department, Menoufia University Hospital and GAMA General Hospital, Saudi Arabia. The study included 30 female patients undergoing breast augmentation of different ages. $\mathrm{Pa}-$ tients with chronic respiratory disorders, smoking, taking regular corticosteroids or anticonvulsant drugs or with systemic connective tissue disorders were excluded from this study.

\section{Pre-operative management:}

At first, detailed history taking was done to record any medical problems such as: Diabetes mellitus, hypertension and any family history for breast cancer or skin disorders. General and local examinations were done after to detect any masses or abnormalities in the breasts. Routine laboratory investigations including complete blood count, kidney and liver enzymes, random blood sugar, prothrombin time \& ratio, hepatitis B, C markers and chest $\mathrm{X}$-ray were performed to complete evaluation of general condition of patients. For patients with suspicion of having masses in their breasts, sono-mammogram and $\mathrm{X}$-ray mammography was done to complete evaluation. A detailed consent concerning operative procedure was taken, in addition to taking standard photos for the breasts preoperatively.

Choosing the appropriate implant size was done properly with the opinion of the patient for the suggested size, most of the sizes chosen were between 350 and $420 \mathrm{mml}$ silicone gel packed.

\section{Surgical procedure:}

The patients were operated under general anesthesia in the supine position, with the operation table bent in a 45 degrees manner and both arms fully adducted in a soldier like position.

Pre-operative marking preceded the operation in which inframammary incision approach was the incision of choice in all operations. A line was marked from the sternal notch down to the xiphoid process to centralize a $3-\mathrm{cm}$ wide cleavage. If the inframammary crease was well established, then the existing crease was used for the incision. But if the nipple-inframammary crease distance was less than $7 \mathrm{~cm}$, the incision line was marked 7 to $7.5 \mathrm{~cm}$ below the nipple. The incision line began 6.5 to $7 \mathrm{~cm}$ from the midline and was about 4.5 to $5 \mathrm{~cm}$ wide.

The pocket marking, on the average, was 14 to $15 \mathrm{~cm}$ in the vertical direction and about 18 to $20 \mathrm{~cm}$ in the horizontal direction depending on the size of the prosthesis, the breast envelope, and the chest wall. The pocket marking did not cross the anterior axillary line.

The nipple-areolar complex was the paramount point used to evaluate the perfection of the position of implant as it should be the most prominent part of the breast. Gaining a full lower pole and an accepted augmented upper pole were properly evaluated as they are important goals for an ideal breast augmentation as well. 
After skin incision, the initial subglandular pocket was made using cutting diathermy up to the lower level of the nipple-areolar complex extending from the junction of the middle and lower third of the sternal fibers of the pectoralis major up and laterally to the anterior axillary line. The subpectoral pocket was reached medially by blunt splitting and lifting of the pectoralis major fibers close to their origin from the junction of the middle and lower third of the sternum. In most patients splitting and lifting was enough to begin forming a wide subpectoral pocket, but some patients had a bulky pectoralis major muscle, which forced me to perform separation of a small part of the middle part of the medial origin of the muscle.

Blunt dissection was performed to achieve the previously marked pocket by introducing index finger in the pocket.

Once the subpectoral pocket was made, a light retractor was introduced in the pocket, and using cutting diathermy, the medial two-thirds of the fibers were split and the lateral third was left intact. This muscle split communicated and changed submuscular and subglandular planes from two planes to one single pocket. The implant, which was a Prefilled, soft, high-profile cohesive gel silicon prosthesis, was introduced in the performed pocket. And the inframammary incision was closed by subcutaneous, intradermal and subcuticular sutures. Usually I did not insert drains after procedure Fig. (1).

\section{Post-operative care:}

The patient was put in a breast compressive garment after operation. And was advised to sleep in a semi sitting position and avoid heavy lifting and exercises directly after the operation.

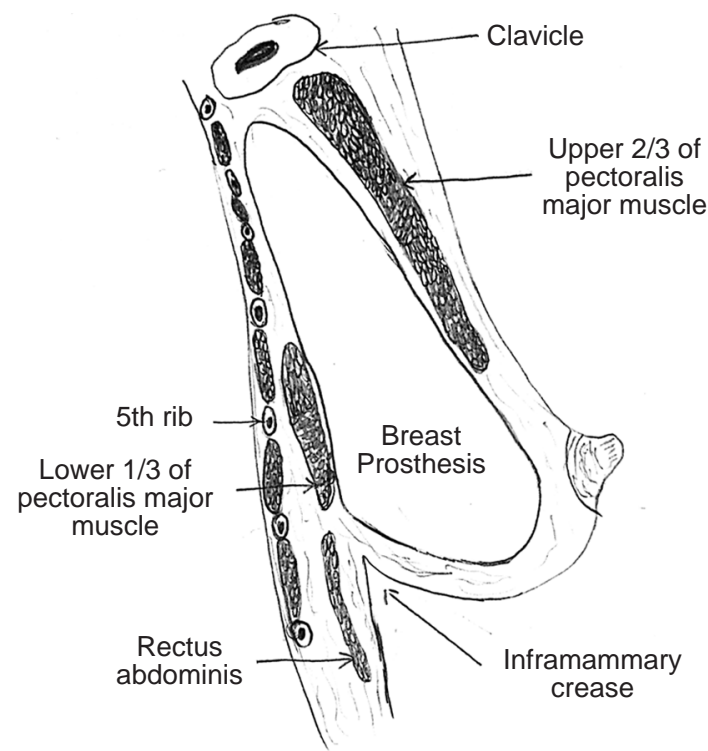

\section{RESULTS}

A total of 30 procedures were performed the period of 5 years using this technique, with a maximum follow-up period of up of 8 months. No hematoma or infection was present with this procedure. No double-bubble deformity, high-riding breast, or superior or superolateral migration was seen.

Nipple sensation was present in all patients and most of them were able to raise their arms above the shoulder and had a reasonable range of shoulder movement before discharge.

Follow-up assessment showed full range of shoulder movements for most of the patients. No muscle contraction or movement associated deformities were seen.

Patients' needs for postoperative analgesia were markedly reduced due to the absence of pectoralis release resulting in quicker recovery. This greatly raised patients' satisfaction towards breast augmentation Figs. (2-5).

Although, 2 patients showed weak satisfaction. The first patient was unsatisfied because of wide cleavage appearance as the patient was very thin, this was corrected after by fat injection in the medial and lower portions of the breast performed 6 months after procedure. The second patient had an obvious difference in volume between the two breasts and after procedures the nipple-areola complex of both breasts where slightly disproportionate in position, but the result was accepted by the patient and no further procedures was done Fig. (6).
Fig. (1): Lateral cross sectional view showing the lower sub-glandular and upper submuscular position of the implant, and its relation to the pectoralis major muscle. 
(A)

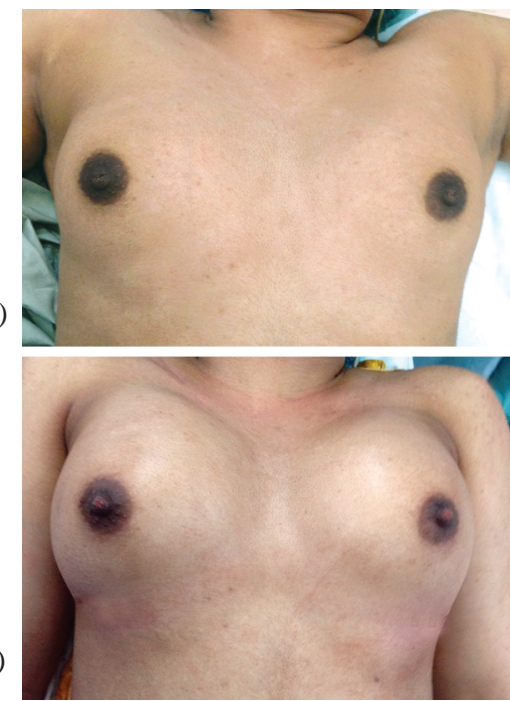

Fig. (2): Anterior view of a middle aged woman with small breasts (a) Preoperative, (b) Postoperative after insertion of a silicon implante sized 350cc.

(A)

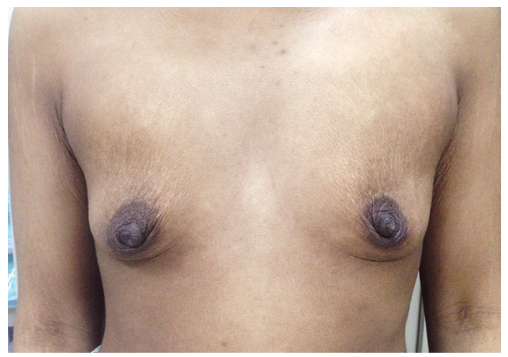

(B)

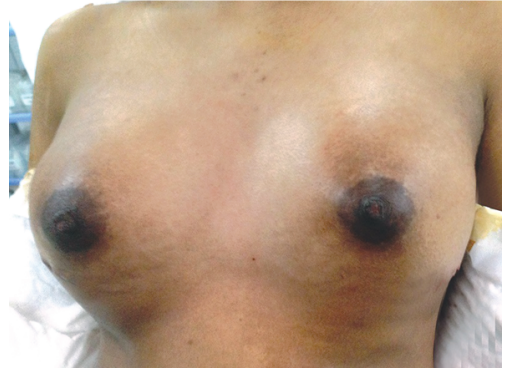

Fig. (4): Anterior view of a 17 years old girl with hypomastia (a) Preoperative, (b) Postoperative after insertion of a silicon implant sized $450 \mathrm{cc}$.

(A)

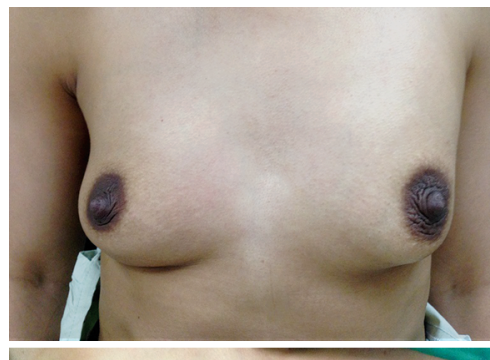

(B)

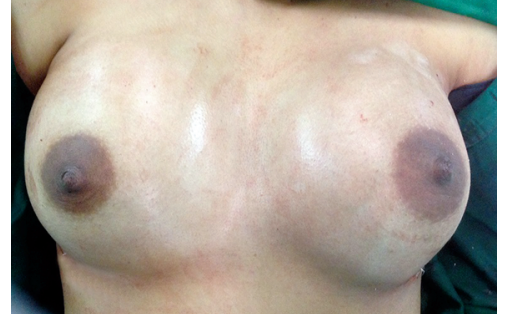

(A)

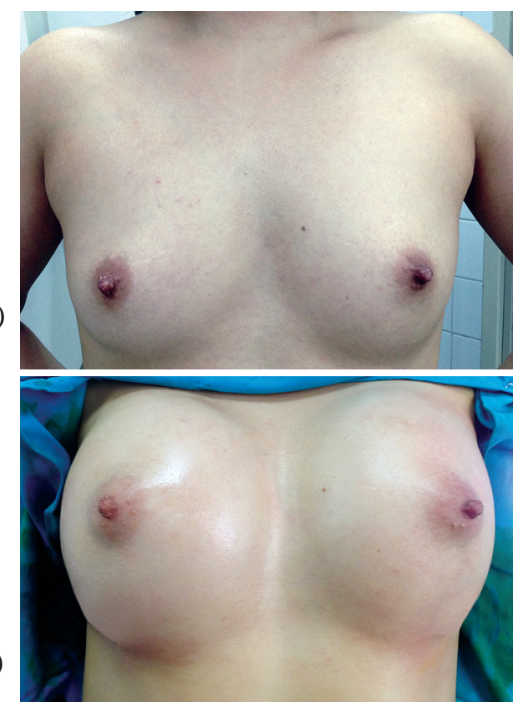

Fig. (3): Anterior view of a young woman with moderate sized breasts (a) Preoperative, (b) Postoperative after insertion of a silicon implant sized 370cc.

(A)

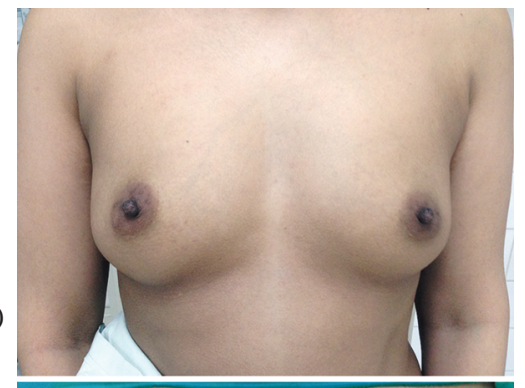

(B)

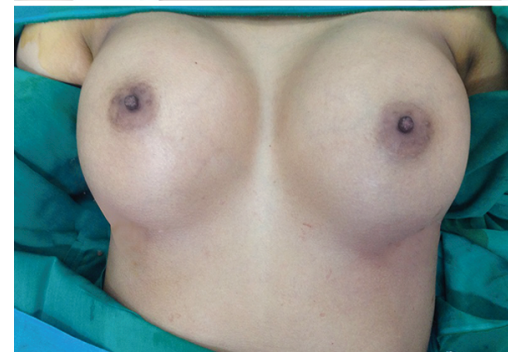

Fig. (5): Anterior view of a middle aged woman with moderate sized breasts (a) Preoperative, (b) Postoperative after insertion of a silicon implant sized $320 \mathrm{cc}$.

Fig. (6): Anterior view of a young woman with moderate sized breasts (a) Preoperative, (b) Postoperative after insertion of a silicon implant sized 350cc. 


\section{DISCUSSION}

To have a successful breast augmentation, it is important to balance the relationship between each type of breasts and its three dimensional continuously changing dynamics. So, when using pectoralis major high splitting technique, prediction of results of breast augmentation will be easy for thin individuals with constricted lower poles or for patients with an excess skin envelope regardless of its thickness.

In thin individuals, we will always find an inadequate skin envelope. The subglandular position of the prosthesis in the lower half when using this technique will give an appropriate enhancement of the lower pole without any restriction by the muscle. In patients with excess envelope, the prosthesis will also fill the lower pole instantly. Use of this procedure for patients with otherwise adequate subcutaneous tissue allows us to insure a long term appropriate result.

Pectoralis major high splitting procedure can be used for type A and minor degrees of type B ptosis with no risk of double bubble deformity, but will never be a good choice for advanced ptosis.

This technique shares most of the dual plane technique objectives [12], but differs from it in that there is no pectoralis muscle release with this procedure as like there is in dual plane technique. Also, the pectoralis major muscle has an anterior and a posterior relationship to the prosthesis in this technique unlike the total anterior position in the dual plane.

For adequate cover of the implant, the upper medial part of the pectoralis major muscle is the only part of the muscle required, and manipulation of the whole muscle as in dual plane technique is unnecessary. Also there is extensive release of costal fibers in duel plane technique which will increase surgical morbidity, time of surgery, postoperative pain, and time of recovery. Muscle cover in the lower pole may restrict breast envelope expansion [8] and the detached muscle will be nonfunctioning and usually does not reduce contraction-associated deformities.

With the splitting procedure, prosthesis cover in the upper medial part of the muscle is gained by splitting the muscle at the junction of the middle and lower third of the sternum and continuing the split along the direction of the fibers laterally and obliquely toward the anterior axillary line.

This will preserve the inferior part of the muscle attached to the costal margin and rectus abdominis and mechanically available for use. Also the two split ends of the pectoralis major meet supralaterally preventing the superior, supralateral, or lateral displacement of the implant in the postoperative period.

To gain an aesthetic cleavage is one of the main targets of breast augmentation, and with a sub muscular pocket, it is determined by pectoralis slips arising from the lower ribs, rectus abdominus muscle, and lower third of the sternum. A better cleavage is achieved by subpectoral pocket procedures, including the dual plane, in which these slips are divided. This division also reduces lateral displacement of the prosthesis and contractionassociated deformities [11].

With the muscle high splitting procedure, sub pectoral pocket entry is made higher than these fibers, preventing the need for extensive release. This achieves an aesthetically pleasing cleavage because the intact upper sternal muscle slips stabilize the bridge of cleavage skin. Also because the sternal fibers at this level are shorter than the costal fibers, with less force of contraction, so contraction associated deformities, as compared with other sub muscular procedures are rarely present.

Passive stretch of skin is prevented by the partial cover of the prosthesis by the muscle in the supra-medial part of the breast. The original attachment of gland and skin to the muscle through the deep fascia remains intact and keeps the upper pole of the breast in a natural shape. Partial muscular cover of the prosthesis usually gives a teardrop appearance when round soft implants are used. This partial cover also hides the edges of the implant, and prevents to observe any abrupt demarcation of the prosthesis under breasts skin.

The muscle intra-operatively in this area will act as a safety to the thin skin when diathermy is used for hemostasis in the sub glandular pocket. Partial cover of the prosthesis in the upper part of the pocket may reduce capsular contracture which is more common in the total sub glandular pocket $[1,2]$.

\section{Conclusion:}

Performing breast augmentation using pectoralis major high splitting technique has provided a versatile pocket option that can be used in most augmentation mammoplasties, and is of great value in giving a three-dimensional improvement in the continuously changing soft tissue dynamics of the breast with quicker recovery periods and better shoulder movement ranges than other techniques. 
As a result, this will decrease morbidity of the procedure and raise satisfaction and favorability of patients toward breast augmentation.

\section{REFERENCES}

1- Biggs T.M., Cukier J.M. and Worthing L.F.: "Augmentation mammoplasty: A review of 18 years". Plast. Reconstr. Surg., 69: 445, 1982.

2- Biggs T.M. and Yarish R.S.: "Augmentation mammoplasty: A comparative analysis". Plast. Reconstr. Surg., 85: 368, 1990.

3- Cronin T.D. and Gerow R.M.:"Augmentation mammoplasty: New "natural feel" prosthesis." In the translation of the Third International Congress of the Plastic Surgery. Excerpta Medica International Congress Series, No. 66 Excerpta Medica, Amsterdam, pp. 41-9, 1964.

4- Dempsey W.C. and Latham W.D.: "Subpectoral implants in augmentation mammoplasty: A preliminary report". Plast. Reconstr. Surg., 42: 515, 1968.

5- Ganong W.F.:" Review of medical physiology". 13 $3^{\text {th }}$ ed. Appleton \& Lange, San Mateo, California, 1987.
6- Graf R.M., Bernardes A., Rippel R., Araujo L.R.R., Damasio R.C.C. and Auersvald A.:" Subfascial breast implant: A new procedure". Plast. Reconstr. Surg., 111: 904, 2003.

7- Keramidas E., Rodopoulou S. and Khan U.D.: "The ballooning manoeuvre in breast augmentation". Plast. Reconstr. Surg., 115: 1795, 2005.

8- Khan U.D.: "Lower pole enhancement in breast augmentation". $6^{\text {th }}$ Croatian Congress of Plastic, Reconstructive, and Aesthetic Surgery, Optija-Rijeka, Croatia, 6-11 October, 2006.

9- Mathes S.J. and Nahai F.: "Classification of the vascular anatomy of muscles": Experimental and clinical correlation. Plast. Reconstr. Surg., 67: 177, 1981.

10- Regnault P.: "Partially submuscular breast augmentation". Plast. Reconstr. Surg., 59: 72, 1977.

11- Strasser E.J.: "Results of subglandular versus subpectoral augmentation over time: One surgeon's observations". Aesth. Surg. J., 26: 45-50, 2006.

12- Tebbetts J.B.: "Dual-plane breast augmentation: Optimizing implant soft tissue relationship in a wide range of breast types”. Plast. Reconstr. Surg., 107: 1255, 2001. 